\title{
In vivo T cell CRISPR screens reveal immunotherapeutic targets
}

Owing to their essential roles in anti-tumour immunity, tumour-specific $\mathrm{CD} 8^{+} \mathrm{T}$ cells are a prime focus of immunotherapy efforts. Although immune checkpoint inhibitors have improved outcomes across many cancers, response rates remain suboptimal, warranting the discovery of novel targets. Using a CRISPR screening approach, a new study led by Sidi Chen now reports the identification of both known and previously uncharacterized regulators of $\mathrm{CD}^{+}$ T cell activity.

The investigators designed and optimized two convergent genome-scale CRISPR screens - an in vivo tumour infiltration screen and an in vitro degranulation screen - to systematically investigate the anti-tumour functions of $\mathrm{CD}^{+}$ $\mathrm{T}$ cells. In the infiltration screen, $\mathrm{CD}^{+} \mathrm{T}$ cells isolated from the spleens and mesenteric lymph nodes of Cas9-mice were transduced with a genome-scale mouse T cell CRISPR library (MKO) - comprising 128,209 gene-specific single guide RNAs (sgRNAs) and 1,000 non-targeting controls - and subsequently injected into mice bearing syngeneic triple-negative breast cancer (TNBC) tumours. Tumour-infiltrating lymphocytes (TILs) were harvested and subjected to high-throughput sgRNA library sequencing and downstream analysis, which revealed sgRNAs targeting genes modulating tumour infiltration. Notably, genes encoding established immunotherapy targets (PD1

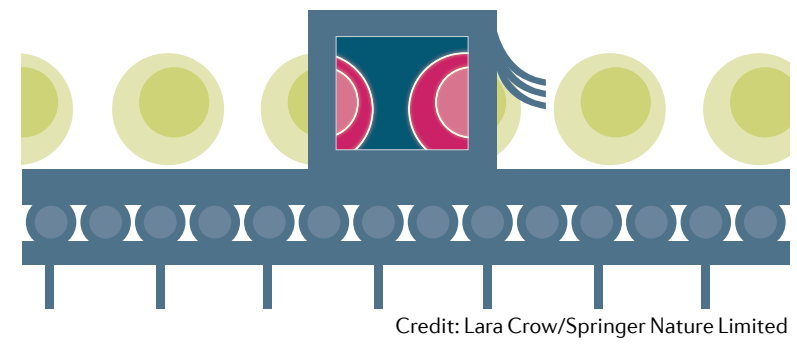

and TIM3) were top hits, providing proof-of-concept of this approach.

In the degranulation screen, MKO-transduced $\mathrm{CD}^{+} \mathrm{T}$ cells were instead co-cultured with mouse TNBC cells, followed by high-throughput sgRNA library sequencing of FACS-sorted $\mathrm{CD}^{+} \mathrm{CD} 107 \mathrm{a}^{+} \mathrm{T}$ cells. Using the same computational approach, potential negative regulators of $\mathrm{T}$ cell degranulation were identified. Importantly, three genes - Dhx37, Lyn, and Odc1 - emerged as common hits between the convergent screens. Functional in vivo experiments showed that, although CRISPR-mediated knockout of $O d c 1$ or $P d c d 1$ (which encodes PD1) in $\mathrm{CD}^{+} \mathrm{T}$ cells reduced tumour growth upon adoptive transfer into mice bearing syngeneic TNBC tumours, Dhx37 knockout led to the greatest increase in anti-tumour efficacy.

As Dhx37 encodes an RNA helicase that has not been previously implicated in $\mathrm{T}$ cell regulation, the authors hypothesized this gene controls lymphocytic function. Indeed, single-cell RNA sequencing (scRNA-seq) of adoptively transferred CD8 ${ }^{+}$TILs revealed major immune-related gene expression changes upon Dhx37 knockout. Furthermore, validation studies using an adeno-associated virus CRISPR knockout assay recapitulated the prior findings of enhanced degranulation in vitro and improved efficacy of adoptive $\mathrm{T}$ cell therapy in the syngeneic TNBC model upon $D h \times 37$ knockout in $\mathrm{CD}^{+} \mathrm{T}$ cells.

Flow cytometry-based immunological characterization of Dhx37-knockout CD8 ${ }^{+}$ $\mathrm{T}$ cells also revealed increased levels of the activation marker CD69, the co-stimulatory receptor $\mathrm{CD} 27$, effector proteins (granzyme B and IFN- $\gamma$ ) and known immune checkpoints (PD1, LAG3 and TIM3), relative to vector-transduced $\mathrm{CD} 8^{+}$ T cells, illustrating that DHX37 regulates $\mathrm{T}$ cell activation and effector function. Consistently, transcriptome profiling of Dhx37-knockout CD8 ${ }^{+} \mathrm{T}$ cells uncovered upregulation of genes associated with $\mathrm{T}$ cell activation and downregulation of gene sets normally expressed in naive or memory T cells. Interestingly, motif enrichment analysis in the promoters of upregulated genes identified the consensus $\mathrm{NF}-\kappa \mathrm{B}$ binding site as the top enriched motif, suggesting that DHX37 functions, at least in part, through interaction with NF- $\kappa B$ components.

In line with the mouse data, DHX37 was upregulated at the mRNA level in activated normal human $\mathrm{CD}^{+}$and $\mathrm{CD} 4^{+} \mathrm{T}$ cells, relative to those in naive states. Moreover, interrogation of scRNA-seq datasets of CD8 ${ }^{+}$TILs from patients with breast cancer showed that, although a high cytotoxic T lymphocyte score was associated with improved overall survival, high $D H X 37$ expression abolished this benefit in patients with luminal-A and TNBC subtypes, highlighting the clinical relevance of the findings.

Overall, the significance of the findings is twofold: the demonstrated utility of CRISPR for immunotherapeutic target identification in vivo warrants its future application in other settings, and the characterization of DHX37 in $\mathrm{CD}^{+} \mathrm{T}$ cells could be foundational for future immunotherapy approaches.

"We plan to further improve the screening system using other viral vector systems, as well as screening in syngeneic (and potentially more sophisticated) models and in more aggressive cancer types such as brain cancers," concludes Chen.

Conor A. Bradley

ORIGINAL ARTICLE Dong, M. B. et al. Systemati immunotherapy target discovery using genomescale in vivo CRISPR screens in CD8 T cells. Cell https://doi.org/10.1016/j.cell.2019.07.044 (2019) 